\title{
Estudio De Los Centros Privados Concertados Religiosos, Desde La Perspectiva De Sus Directores Titulares, En El Contexto Multicultural De La Ciudad Autónoma De Ceuta
}

\section{Arturo Fuentes Cabrera Arturo Manuel Fuentes Viñas}

Universidad de Granada

doi: 10.19044/esj.2016.v12n1p40 URL:http://dx.doi.org/10.19044/esj.2016.v12n1p40

\begin{abstract}
World population is constantly changing. That fact offers contexts where the multiculturalism is more important. Ceuta, a Spanish city located in the North of Africa, is one of the best examples of this multiculturalism. In this context, it is interesting to study the development of the education and its staging. In this research, some schools which form the educational offer in the city of Ceuta were taken as models. We were attracted by the idea of carrying out a detailed research about the private and the receiving state subsidy schools which a religious nature, in order to know their features, the connections inside and outside of the centre and the maintenance of them, among other factors. To get that, a quantitative research with surveys has been carried out. At the same time, the semi-structured interviews have provided some qualitative data. In this research, the results show how "difficult" is the survival of these institutions and the preservation of the thinking of all of them in the multicultural context aforementioned. In spite of the complex reality, we conclude by affirming that these institutions are highly regarded in the city of Ceuta.
\end{abstract}

Keywords: Education, multiculturalism, private teaching

\section{Resumen}

La población mundial está en constante cambio, eso provoca contextos donde cada vez la multiculturalidad se hace más presente. Ceuta, ciudad española al norte de África, es uno de los mejores exponentes de esa multiculturalidad. En ese contexto, es interesante estudiar el desarrollo de la educación y su puesta en escena. En este trabajo, se ha procedido al estudio de una parte de los centros educativos que forman parte de la oferta 
educativa de la ciudad de Ceuta. Nos ha despertado el interés, realizar un estudio pormenorizado de las instituciones privadas y concertadas, que además poseen carácter religioso, que mantienen centros en la ciudad, para conocer sus pormenores, las relaciones dentro y fuera del centro o el sostenimiento del mismo entre otros factores. Para ello, se ha realizado un estudio cuantitativo a través de cuestionarios, contrastándolos con entrevistas semi-esctructuradas que nos han arrojado datos de carácter cualitativo. En este trabajo se muestran los resultados que nos demuestran lo "difícil” de la supervivencia de estas instituciones $y$ sus idearios en el contexto multicultural al que nos referimos. A pesar de su complicada realidad, concluimos que estas instituciones gozan de buena salud y su prestigio es amplio en la ciudad de Ceuta.

Palabras clave: Educación, multiculturalismo, enseñanza privada

\section{Introducción}

Ceuta, es una ciudad fronteriza situada al norte de África donde conviven habitantes de cuatro culturas distintas: cristianos, musulmanes, judíos e hindúes. Ello es lo que le confiere un acentuado carácter multicultural, donde reside su riqueza cultural. Al ser un enclave tan singular, está bañada tanto por el Mar Mediterráneo en una de sus costas, como por el Océano Atlántico en otra. Posee una frontera terrestre con Marruecos, con las regiones de Tánger- Tetuán, lo que le confiere ser una de las de mayor desigualdad existentes a ambos lados de la misma. Ceuta ha sido considerada desde la antigüedad como Plaza Mayor, Ciudad Presidio, Plaza Fronteriza y, durante el régimen de Franco, Plaza de Soberanía (Martín, 2012). Por su enclave, ha formado parte de la estrategia de cuantas civilizaciones quisieron conquistar parte del territorio ibérico y africano. La Ciudad posee un status político de Ciudad Autónoma que el Estado le confirió en el año 1995, mediante un Estatuto especial de Autonomía que regiría sus destinos desde ese momento hasta la actualidad. La Transitoria V de la Constitución Española, da vía libre a las ciudades de Ceuta y Melilla para convertirse en Comunidades Autónomas. Sin embargo, debido a las presiones marroquíes, no fue hasta la fecha antes mencionada cuando se da luz verde al Estatuto de Autonomía.

Ceuta, pues, se constituye como un ente autonómico no equiparable a una comunidad autónoma. Ceuta tiene transferidas, por parte del Gobierno, algunas competencias en materias políticas, siendo otras como la Educación, competencia directa del Estado y los poderes públicos estatales, teniendo Delegación en la Ciudad Autónoma. Además, a raíz de la concesión de la autonomía parcial, se duplicaron los cargos en la ciudad, ya que todos los cargos municipales pasaron a convertirse a su vez en autonómicos. Se 
produce por esta causa que el alcalde de la ciudad, sea considerado también como Presidente de la misma, debido al carácter dual de la institución que la rige.

En las últimas encuestas e informes educativos, Ceuta se encuentra de las últimas en cuanto a índices educativos se refiere, siendo el fracaso escolar uno de los máximos exponentes de la ciudad en la actualidad. El abandono escolar temprano supera la media nacional con un 29,5\%.

Para realizar este trabajo, se ha decidido estudiar las tres instituciones privadas religiosas, por considerar precisamente interesante su "supervivencia" en el sistema concertado y en el contexto en el que se encuentran. Los tres centros que han decidido estudiarse han sido los Centros Privados Concertados religiosos que existen en la Ciudad Autónoma de Ceuta en la actualidad: El Colegio "La Inmaculada”, el Colegio "San Agustín” y el Colegio “Santa María Micaela”.

El colegio "La Inmaculada”, cuya titularidad pertenece a la orden de las misioneras de la Inmaculada Concepción, es uno de los colegios con más antigüedad de la ciudad de Ceuta, y que goza de mayor prestigio en la misma.

La fundadora de la orden, figura clave en su desarrollo, es María Alfonsa Cavín. Nace el 17 de noviembre de 1816, inscrita en el registro civil con el nombre de Louise- Félicie, provenía de una familia dedicada a la metalurgia establecida en Montbèliard, Franco Condado. Su padre, al igual que sus antecesores, se dedicaba al trabajo del metal, ocupando puestos de responsabilidad en las distintas fraguas que trabajó, eso suponía que su nivel económico era desahogado y le permitió adquirir algunas propiedades; se podría decir que su familia pertenecía a la pequeña burguesía de la época.

$\mathrm{Su}$ familia era de profundas creencias religiosas, permaneciendo fieles a la Iglesia Católica pese a desarrollar su vida en ambientes propicios a otros tipos de creencias. Fieles a sus convicciones y a sus tradiciones, pero al mismo tiempo (...) mentalidad de minoría cerrada (Lozano, J.M.,1979, p. 10).

Hoy día la Orden Religiosa cuenta con congregaciones por medio mundo dedicadas a la Enseñanza, a la asistencia y a las misiones, según lo ordenado por su fundadora. En Andalucía y Norte de África cuenta con 18 congregaciones, en Cataluña con 10, en África un total de 8, en América del Sur (Argentina, Colombia, Paraguay, México y Venezuela) 24 y en Italia, 1.

El Colegio La Inmaculada Concepción de la ciudad de Ceuta es el centro privado religioso más antiguo instalado en la hoy Ciudad Autónoma, tiene detrás una larga trayectoria docente y un arraigo importante en el tejido social de la población, siendo muchas las generaciones de jóvenes, fundamentalmente chicas, las que se han formado en los distintos establecimientos con que ha contado la Orden desde que comenzara su labor 
docente un 24 de enero de 1887, pero la instalación de la Orden religiosa no fue fácil, impulsada por una serie de personajes claves para la historia de Ceuta en aquellos tiempos. Poca documentación hay al respecto salvo lo que ha servido para intentar recomponer la interesante historia de este centro educativo de marcado carácter religioso, pero de un prestigio importante en la ciudad. Estos documentos son los originales que constan en el Archivo Catedralicio de la Ciudad Autónoma de Ceuta y la Crónica General de los ciento veinticinco años de historia de este Colegio, editado a nivel interno por el propio Colegio para la celebración de este aniversario.

Actualmente, el Colegio es un Centro Concertado de Infantil, Primaria y Secundaria atendiendo aproximadamente 900 alumnas y alumnos, ya que desde hace varios años se convirtió en un Colegio mixto. El Claustro de profesores está compuesto en su mayoría por seglares.

El objetivo fundamental es "la formación integral de sus alumnos en los aspectos humanos, sociales y religiosos". Todo ello a base de una programación coordinada, por medio de un contacto directo con los alumnos. La acción educativa de la escuela concepcionista promueve el cultivo de las costumbres y la cultura del país en lo social, político y eclesial a fin de dar respuestas adecuadas a las necesidades de los educandos.

Actualmente es una Institución perfectamente consolidada, como parte integrante del tejido social de la ciudad, que ha sabido formar a lo largo de su difícil existencia generaciones tras generaciones de niñas y jóvenes ceutíes, tras los pasos del Evangelio, con la fuerza que supo imprimir en su día la M. Alfonsa a sus hijas.

El siguiente de los centros que ha sido objeto de estudio de este trabajo, a raíz de lo expuesto por su director ha sido el Colegio San Agustín, centro que este año celebra el centenario de su fundación y que tiene a San Agustín como centro de sus ideales y modelos de enseñanza.

Aurelius Augustinus nace el 13 de noviembre del año 354 en Tagaste, cuando reinaba el emperador Constancio II. Este pequeño pueblo, se encontraba en la provincia romana de Numidia, África del norte, en el valle del oued Medjerda. Una vida algo díscola, dio paso a una vida contemplativa y religiosa. A partir de ese momento, Agustín se convertirá en una figura importante para el desarrollo teórico de la religión. Participa en todos los concilios a los que tiene acceso, comienza sus confesiones y sus tratados, sus escritos y sus sermones. Agustín, tras designar a Heraclio como su sucesor en el obispado, fallece el 28 de agosto en Hipona, que estaba siendo asediada por los vándalos.

La orden agustina, ha ido creciendo con el paso del tiempo, siendo su vida educativa rica en favores a la educación, y uno de los bastiones principales de su fortaleza. Su historia educativa en Colegios les permite ofrecer a la sociedad una educación integral, para que los alumnos 
perfeccionen todas sus potencialidades, un sistema de coeducación, una gestión escolar participada por toda la Comunidad Educativa, según lo establecido por las leyes de enseñanza, y una calidad educativa propia, inspirada en los siguientes valores agustinianos:

- Un progresivo aprendizaje que conduce al conocimiento siempre creciente de toda la realidad, especialmente del hombre y de Dios.

- $\quad$ Una sincera y noble apertura a todos los seres humanos para construir una sociedad más fraterna y solidaria.

Desde la inauguración del Protectorado español en Marruecos, en 1912, la Provincia Agustina de Filipinas, comenzó a estudiar y hacer firmes las posibilidades de lograr una fundación en tierras norteafricanas, a falta de un enclave concreto. Por una parte, con la puesta en marcha de esto se lograba llenar un vacío sentimental, ya que San Agustín era natural de Tagaste y por tanto norteafricano, quedando pocas posibilidades de instaurarse en ese continente si no era en Ceuta, y por otro lado, la necesidad de encontrar un punto geográfico en el cual los religiosos de la Provincia pudiesen cumplir con sus deberes militares prosiguiendo en el cumplimiento de los eclesiásticos.

En abril del año 1913, se consigue por fin que aceptasen la petición de la instauración en Ceuta de la Comunidad, resuelta por el Marqués de Vadillo el 7 de mayo de 1914. Más tarde, el 10 de junio, llegaría la licencia del Obispo, don José María Rancés y Villanueva, y tres días más tarde el ofrecimiento de la Iglesia de San Francisco, que regentan los Padres Agustinos desde entonces hasta la actualidad.

Los primeros religiosos de la Orden llegan a Ceuta en el mes de junio. En agosto ya se habían firmado las escrituras del terreno y en octubre de ese mismo año, 1914, ya habían comenzado las obras en el solar, con los movimientos de tierras oportunos. Desde que el Provincial al que hemos dedicado las páginas anteriores, el P. Bernardo Martínez, encargara el mantenimiento de la Iglesia de San Francisco y la construcción del colegio, no se escatimó en esfuerzos para hacer realidad lo ideado por la Provincia Filipina en Ceuta. Hasta el día de hoy, el Centro "San Agustín”, ha venido ofreciendo una educación de calidad y basada en sus ideales.

Actualmente cuenta con todas las instalaciones necesarias para desarrollar su actividad educativa: aulas de clases amplias, aulas complementarias de refuerzo y apoyo, aula de informática, laboratorios de Física, Química y Ciencias Naturales, biblioteca, patio de recreo y deportes, vestuarios...

La Provincia de España de la Orden de San Agustín, como titular, define la identidad y el estilo educativo del Colegio y tiene la misma responsabilidad del mismo ante la sociedad, la administración educativa competente, los padres de alumnos, el profesorado, los alumnos y el personal 
de administración y servicios (LODE, arts. 21 y 22; S.T.C. 85-II, 8, 10, 20).

El nivel socioeconómico-cultural de las familias es, preferentemente, de clase media. Predominan los padres con estudios superiores, profesiones liberales y funcionarios. El Centro, en los niveles obligatorios, está acogido al régimen de conciertos regulados en el Título IV de la Ley Orgánica del Derecho a la Educación, en el Reglamento de Normas Básicas sobre Conciertos Educativos, aprobados por el Real Decreto 2377/1985, de 18 de diciembre, y en sus normas de desarrollo en los niveles de Educación Primaria y Educación Secundaria Obligatoria.

El último de los centros que ha sido objeto de estudio de este trabajo ha sido el centro "Santa María Micaela”, cuya titularidad ostenta las hermanas Adoratrices, como cariñosamente se les conoce en nuestra ciudad, y cuya fundadora, ha sido figura fundamental en su desarrollo.

La fundadora de la Orden religiosa que daría origen a uno de los centros de estudio de este trabajo, de nombre Micaela, nació en plena guerra de la Independencia, más exactamente el primer día del año de 1809, su nombre natural era Micaela Desmaisières López Dicastillo y Olmedo, de título nobiliario, vizcondesa de Jorbalán. Esta joven recibió a lo largo de su niñez y juventud una educación propia de su rango social.

Provenía de una familia de militares de alto rango en la corte española de Carlos IV; de hecho sus padres se conocieron en el Palacio Real ya que su padre era un joven oficial que pertenecía a la Guardia Real que custodiaba al Rey, y su madre era camarista de la Reina, destinada al cuarto de la Infanta Doña María Isabel. Su boda tuvo que ser autorizada expresamente por el mismísimo Rey, quien dotó a la camarista para la boda con 500 ducados.

Se resalta lo anterior porque nos indica el origen nobiliario de nuestra protagonista. Ella llegó al mundo cuando ya habían llegado a su familia cuatro hermanos. A pesar de todas estas circunstancias que hacen mucho más extraordinaria su vida al darle un giro espectacular con un abandono de todo lujo para dedicarse por entero a los necesitados, dando lugar a una orden que se ha convertido en fundamental para la historia de la Iglesia y de la Educación.

El crecimiento de la Orden ha sido muy relevante, manteniéndose vivo el espíritu "micaeliano" en todas sus obras, que han sabido respetar todas las sucesoras de la fundadora, ya que desgraciadamente esta murió joven (24 de agosto de 1865) cuando acudió a Valencia a socorrer a las religiosas y colegiadas de su casa, aquejadas de una epidemia de cólera que estalló en la ciudad. Independientemente de los Colegios, las Adoratrices han fundado muchas “casas hogar”, para la formación de las jóvenes, sobre todo, en zonas o países necesitados, es el caso de Marruecos, donde dependiente del Colegio de Ceuta se han fundado casas en poblaciones de pocos 
habitantes del vecino país, próximas a Ceuta. En ellos se les imparte los conocimientos elementales de nuestro modelo educativo, corte y confección, cocina, etc.

Ochenta años de existencia corroboran la importancia que para esta ciudad ha tenido el Instituto de Religiosas del Santísimo Sacramento (Adoratrices). Su labor desarrollada durante todos estos años va más allá de los meramente pedagógico, para intentar ponera esta obra su auténtico valor no podemos desgajarla y juzgarla de forma parcelaria, sin tratar de entender el conjunto del modelo social que esta Orden ha venido proponiendo a la sociedad ceutí a través de sus aulas y casas de acogidas; toda acción realizada ha ido y va impregnado de un modelo ideológico basado en la "solidaridad", principio y fin de su existencia, que aún hoy está tan vigente como aquel primer día que la M. Sacramento creó una casa de acogida para prostitutas en la calle San Lorenzo de Madrid.

La historia de este Colegio es muy rica y llena de acontecimientos, desde su casual implantación en la ciudad han sido muchas jóvenes, tanto de Ceuta como llegadas de la Península y del Protectorado de Marruecos las que han recibido algún tipo de formación en sus aulas, a veces improvisadas por la falta de recursos. Esas jóvenes, en su mayoría eran personas necesitadas o con otros tipos de problemas que acudían cada día al socorro de las Hermanas de la Congregación; evidentemente, el paso del tiempo han hecho cambiar el planteamiento de su labor solidaria, pero lo que no ha cambiado nunca es su "carisma" que sigue siendo un referente que impregna toda la acción, tanto educativa como de ayuda.

Hoy en día, el Colegio Santa María Micaela sigue gozando de una buena salud y un gran prestigio en la Ciudad Autónoma de Ceuta. Sigue albergado en el edificio que fue cedido a las adoratrices en la Barriada de "El Sardinero", siendo de carácter privado concertado. Forma un sistema Multisite junto a los otros once Centros de Adoratrices de España.

Cuenta actualmente con una plantilla docente compuesta por 35 profesores, con un equipo directivo compuesto por una Directora Pedagógica, una Directora de Educación Infantil y de Primaria, un Jefe de Estudios de la Educación Secundaria Obligatoria, una secretaria y una administradora del centro.

\section{Metodología}

En cuanto al análisis descriptivo de los centros actualmente se ha utilizado una metodología ecléctica que resulta ser integradora y complementaria, esto es a través del estudio cuantitativo y cualitativo. El objetivo de este trabajo ha sido "Conocer, describir y valorar el origen, desarrollo y estado actual de la enseñanza privada religiosa católica reglada en la Ciudad Autónoma de Ceuta, a través de las opiniones de sus 
directores titulares". Además, se han establecido otras metas como son:

- $\quad$ Análisis de aspectos derivados de las relaciones entre la Patronal y el Equipo directivo pedagógico del centro.

- $\quad$ El ejercicio que le obliga la responsabilidad del cargo.

- $\quad$ El perfil adecuado para la figura del Director Pedagógico.

- Valoración del actual sistema de Conciertos que sostiene, en gran medida, este tipo de establecimientos educativos.

- $\quad$ Los problemas derivados de la financiación.

- $\quad$ Visión sobre el actual sistema de Admisión de alumnos implantado por la Administración Educativa para los centros sostenidos con fondos públicos.

- $\quad$ Los Recursos de los que dispone el centro educativo.

- $\quad$ La Formación del profesorado.

- Aspectos derivados de las relaciones con las familias, la Administración Educativa y otras Instituciones locales con las que guardan estrecha relación.

- $\quad$ Valoración de la convivencia en el centro.

- $\quad$ Las relaciones entre la Entidad Titular y el Equipo directivo.

- Grado de aceptación e implicación a los distintos cambios legislativos producidos en los últimos tiempos en nuestro Sistema Educativo y que afecta directamente a estas Instituciones.

A la hora de realizar este trabajo, se tuvo la idea clara de que, quien mejor podía darnos a conocer el estado actual de la cuestión a analizar, serían sin duda los protagonistas de los centros educativos que son objeto de estudio. Es por ello que se decidió acudir a los propios centros educativos para que tanto los Titulares de los mismos nos mostraran de primera mano sus inquietudes, sus percepciones y sus valoraciones. Se ha establecido una doble vertiente: por una lado cuestionarios ad-hoc, personalizados para la investigación y, por otra parte, una entrevista semi-estructurada para apoyar y triangular los resultados. Han participado los tres centros a los que se ha acudido, por lo que la muestra ha sido de 3 personas y la participación del 100\%. Esta muestra se refiere a los tres Directivos Titulares de los Centros Educativos que hemos tenido ocasión de estudiar.

El cuestionario fue sometido a juicio de expertos, especialmente de D. Manuel Lorenzo Delgado para su validación. A la hora de analizar los cuestionarios, los resultados nos han ofrecido un valor del Alfa de Cronbach de 0,973, por lo que queda demostrada su fiabilidad. En relación a la recogida de datos cualitativos, se formó un grupo de discusión, que es una técnica que trabaja con el habla, se fija en la subjetividad y el orden social, por tanto lo dicho y lo excluido en el grupo se explican recíprocamente, según Arnal y otros (1994). Se realizó una estructura semi-estructurada, que 
atendía a parecidas cuestiones que englobaban todos los ítems del cuestionario, para la triangulación de estos y poder extraer conclusiones determinadas.

Los Directivos Titulares de los centros educativos que hemos tenido ocasión de entrevistar, han sido 1 hombre y 2 mujeres, de más de 41 años de edad que llevan en el cargo de 0 a 4 años y con más de 13 años en la docencia, por lo general. Con los datos recabados en los centros se llevó a cabo un examen exhaustivo y puramente estadístico de los datos de carácter cuantitativo y de los datos de carácter cualitativo, dando forma a la investigación.

Los instrumentos que hemos utilizado para recabar datos e información a lo largo de todo este trabajo que aquí se presenta, han sido de diversa naturaleza e índole, por lo que podemos afirmar que posee una metodología de investigación mixta. Han sido utilizado los siguientes instrumentos:

- $\quad$ Cuestionarios para los directores titulares de lo centros educativos religiosos.

- Entrevista semi-estructurada con el fin de triangular los datos obtenidos en los cuestionarios a directivos titulares, con los mismos.

El cuestionario fue sometido a juicio de expertos, especialmente de D. Manuel Lorenzo Delgado para su validación. En este sentido, Fox (1981), manifiesta que la validez de contenido es la técnica más apropiada cuando se trata de validar cuestionarios. Considera que nos informa de la representatividad del contenido del cuestionario y sí mide lo que pretende medir.

El cuestionario dirigido a los Directores Titulares de los centros educativos atendía a ámbitos tales como:

- Datos sociológicos.

- $\quad$ Datos sobre el cargo y su tarea personal.

- $\quad$ Datos sobre la gestión del centro y financiación del centro.

- $\quad$ Datos respecto al profesorado del centro.

- Datos respecto a las relaciones dentro del centro.

- $\quad$ Datos sobre la actualidad educativa y la normativa vigente.

En relación a la recogida de datos cualitativos, se formó un grupo de discusión, que es una técnica que trabaja con el habla, se fija en la subjetividad y el orden social, por tanto lo dicho y lo excluido en el grupo se explican recíprocamente, según Arnal y otros (1994). Se realizó una estructura semi-estructurada, que atendía a las siguientes cuestiones.

- $\quad$ ¿Cómo es la relación entre la Entidad Titular y el Equipo Directivo del Centro?

- $\quad$ ¿Considera que el proceso de elección del Director Pedagógico es el 
más adecuado?

- ¿ ¿Qué aspectos valoran más en la elección de un profesor como Director Pedagógico?

- $\quad$ ¿Qué opinan del Sistema de Concierto Educativo al que está acogido el Centro?

- ¿ ¿ ¿ con respecto a la financiación para el sostenimiento del Centro?

- $\quad$ Sobre el sistema de admisión de alumnos al que obliga la Administración educativa ¿Cuál es su punto de vista?

- $\quad$ ¿Cree que es correcta la forma de contratación del profesorado por parte de la Entidad Titular?

- ¿ ¿Son suficientes los recursos de que dispone el Centro Educativo?

- $\quad$ ¿Cómo es la oferta formativa del profesorado?

- $\quad$ Descríbame la relación de la Entidad Titular con las familias de los alumnos/as.

- ¿ ¿La convivencia es buena entre los distintos integrantes de la comunidad educativa?

- ¿ ¿ ¿ la relación con la Administración?

- $\quad$ ¿Cómo es la colaboración entre la Entidad y el Equipo Directivo del Centro?

- ¿ ¿Qué opina sobre los sucesivos cambios legislativos en materia educativa producidos los últimos años en nuestro país? ¿Cuál es su postura?

\section{Resultados}

A lo largo de la elaboración de este artículo, tanto en su fundamentación teórica como en la posterior recogida y análisis de la información relevante para nosotros, creemos que hemos llegado a cumplir con éxito el objetivo general planteado así como de los objetivos específicos derivados.

La intención de todo este trabajo no ha sido otra que realizar una panorámica descriptiva actualizada de la enseñanza privada religiosa católica reglada en la Ciudad Autónoma de Ceuta, con todas sus sombras y luces con el fin de conocer todos aquellos factores que influyen en su desarrollo y que conforman su propia identidad.

La valoración posterior de los datos obtenidos a través de los cuestionarios, no pretende en ningún momento otra cosa que abrir nuevas vías de comprensión y colaboración en la tarea educativa entre las distintas Administraciones y organismos a nivel externo; y servir de ayuda para implementar nuevas dinámicas de trabajo y mejorar las ya existentes entre las plantillas de este tipo de centros a nivel interno.

Respecto al objetivos general de "Conocer, describir y valorar el origen, desarrollo y estado actual de la enseñanza privada religiosa 
católica reglada en la Ciudad Autónoma de Ceuta, a través de las opiniones de sus Directores Titulares", creemos haberlo cumplido a través de todo el trabajo realizado, ofreciéndonos una “fotografía” esclarecedora de su estado actual.

Con respecto a las distintas características del Director Titular, el perfil encontrado, sus actuaciones, relaciones con la comunidad educativa (profesores, familias, representantes de las distintas administraciones, etc.) así como sus valoraciones sobre los cambios legislativos en materia educativa, podríamos decir que nos encontramos con un perfil "tradicional" en donde esta figura normalmente es elegida a partir de su experiencia, madurez personal y sobre todo identificación con una serie de valores vividos y compartidos por las distintas órdenes religiosas que conforman este tipo de Centros.

Predominan las mujeres (en este caso, se confirma que en órdenes religiosas femeninas aparecen Directoras y en la masculina director, aunque no podemos aseverar que este sea el motivo exclusivo) como directoras titulares. Todas con una edad intermedia y con experiencia dilatada de servicio.

Se trata de personas comprometidas, como decíamos, que utilizan las relaciones humanas como herramienta significativa de integración en el Centro, y de cohesión a través de la adhesión a los valores ofertados en sus Idearios y programaciones. Sin embargo, mantienen unas líneas claras de preservación de los intereses y necesidades de la organización sin menospreciar las necesidades de la comunidad en la que se hayan inmersos, sino intentando potenciar su desarrollo.

La responsabilidad del cargo, les obliga a trabajar en este complicado equilibrio entre calidad educativa, gestión adecuada de los recursos, clima organizacional y una buena oferta educativa para todos. Es por ello, que mantienen cauces de comunicación y colaboración adecuados con la Patronal y el resto de docentes.

La relación con el equipo directivo destaca por ser buena $y$ cohesionada como grupo a la hora de realizar un trabajo colaborativo, al igual que con los padres y madres, y docentes; sin embargo, es remarcable la necesidad de mejorar algunos aspectos referidos a la percepción de algunos sectores sobre cómo son valorados, y su implicación en las distintas actividades o tareas a desarrollar.

Por ejemplo, en la convivencia en el Centro o la participación de las familias en las iniciativas planteadas a nivel formativo o recreativas. La participación de las familias como un elemento significativo en el desarrollo educativo de sus hijos es un aspecto que creen debe ser mejorado. Programas como los desarrollados para evitar el acoso o trabajar la igualdad de género y 
la prevención de conductas disruptivas obtienen mejores resultados cuando las familias colaboran con los centros educativos.

Las relaciones con las distintas Administraciones siguen una línea de normalidad en lo referente a las diferentes comunicaciones y colaboración con estas. Sin embargo, existe una percepción de mayor exigencia en este tipo de centros a la hora de cumplir con las distintas disposiciones, normativas o rendición de cuentas a nivel de beneficios.

El actual sistema de conciertos es visto como adecuado y totalmente necesario para su subsistencia. Los cambios en las concepciones sobre este tipo de centros en los últimos años, y el cambio en las políticas educativas y presupuestarias que les afectan, están ocasionando que en no pocos Centros existan dificultades para gestionar adecuadamente las contrapartidas provenientes de las Administraciones.

Estas contrapartidas son insuficientes para gestionar adecuadamente o proveer nuevos recursos tanto humanos como materiales o formativos, a pesar de que en los cuestionarios realizados nos hablen de unos balances equilibrados. Es el caso por ejemplo de las instalaciones para la realización de las distintas reuniones o actividades, entre ellas las relacionadas con las Nuevas Tecnologías.

El profesorado demanda formación específica en este sentido y, sin embargo, un escaso porcentaje posee las instalaciones para desarrollarlas adecuadamente. En algunos casos, se demandan mejores espacios o la creación de estos, como es el caso de las AMPAS que no poseen un lugar donde poder realizar sus tareas.

Otra fuente de conflicto detectada es el sistema de admisión de alumnos a los Centros. Se considera que este sistema a seguir no es el idóneo ya que genera numerosas irregularidades y problemas debido a la manipulación de los datos por parte de las familias con el fin de conseguir que sus hijos e hijas consigan los puntos de baremación suficientes para ingresar en los Centros deseados.

Es por ello que es preciso, como hemos mencionado en las posibles líneas futuras de trabajo, llevar a cabo encuentros con las distintas partes implicadas con el fin de buscar soluciones alternativas idóneas para todos en las que se atienda el derecho de los padres y madres a la libre elección de Centro sin mermar la equidad en educación.

Por otra parte, como representantes de la Entidad a la hora de seleccionar personal podríamos decir que se centran significativamente en los valores y personalidad del candidato (que sea una persona responsable, sincera, ética, con valores...) al mismo tiempo que con la formación pertinente o al menos necesaria para ejercer el cargo por ejemplo de Director Pedagógico o maestro. 
El perfil demandado por este tipo de entidades y por los Directores titulares para Director Pedagógico, responde al de una persona capaz de interaccionar socialmente de manera positiva y mantener unas buenas relaciones sociales con la Patronal y el resto de implicados en la labor educativa. Otras características como unas adecuadas competencias de planificación, gestión y pedagógicas son tenidas en cuenta en menor medida, sin ser menospreciadas.

De igual forma, la contratación del resto del personal es vista desde un punto de vista restrictivo a la hora de seleccionar quién cubre las vacantes de acuerdo con los procesos administrativos de las distintas administraciones, por lo que se demanda una mayor libertad en este sentido. Como veíamos anteriormente, los Directores basan parte de sus decisiones en el compromiso de los aspirantes a desarrollar los valores que comparten.

Una vez que los aspirantes forman parte de la plantilla, los Directores consideran que la formación que se realiza desde la Entidad Titular es la más adecuada y pertinente para sus necesidades.

Con respecto a la aceptación e implicación de los Directivos en los cambios legislativos, y en concreto en la implantación de la LOMCE, podemos concluir que a pesar de estar motivados e informados sobre los distintos cambios, sus expectativas de mejora del sistema educativo no son muy positivas.

Los Directores de estos centros consideran que se han producido demasiados cambios en función del partido en el gobierno con la consiguiente merma en la calidad y eficacia del modelo educativo del país. Creen que es preciso llegar a un consenso imprescindible actualmente entre todas las partes y tendencias (escuela pública, concertada y privada) de tal forma que sirva realmente para implementar medidas correctoras y hacer que la nueva ley educativa (la LOMCE) sea algo más que otro cambio.

Con todo lo mencionado hasta el momento, estos directivos poseen un perfil motivado y reconocido socialmente según su percepción, que les hace mantener unos niveles aceptables de satisfacción a pesar de las dificultades surgidas en el trabajo y seguir implicándose en las tareas diarias.

\section{Conclusion}

Se pretende en este apartado realizar unas conclusiones finales con respecto a este trabajo. Cabe destacar que no existen apenas investigaciones sobre el tema que se ha trabajado en este artículo, por lo que esperamos que estas conclusiones y posteriores líneas y propuestas futuras de investigación sirvan como "punto de partida" para investigaciones futuras de esta naturaleza o parecida.

A modo general podemos extraer las siguientes conclusiones finales: 
- $\quad$ La enseñanza privada ha supuesto el principal pilar en el que se ha sustentado la educación durante casi toda su historia. No fue hasta que los Estados intervinieron en la misma, cuando se comienza a mostrar un interés por un sistema que no fuese el privado.

- $\quad$ Si existe una protagonista indiscutible de ese tipo de enseñanza es, sin duda, la Iglesia Católica que durante siglos ha mantenido la tradición educativa a la que está obligada, como servicio a los demás que es, por sus ideas y pensamientos.

- Los tres centros educativos que subsisten en la ciudad, amparados bajo la titularidad de la Iglesia, están plenamente integrados en el "paisaje” de la Ciudad de Ceuta y gozan de un prestigio bastante alto.

- $\quad$ Los idearios de los centros privados y concertados se han adaptado a lo que la ley exige de los centros, lo que no ha hecho más que menguar la exposición de estos idearios.

- Los centros privados concertados, se han impuesto a la modalidad completamente de carácter privado, lo que ha facilitado la subsistencia de muchos centros pero, a su vez, también la pérdida de identidad de algunos de ellos.

- Que los directores titulares de los centros educativos cuentan con grandes conocimientos de la institución de la que son responsables y muestran una conexión permanente con sus equipos directivos y docentes.

Sería conveniente seguir trabajando en estos temas que aquí presentamos. Como futuras líneas de actuación, proponemos las siguientes vías:

- $\quad$ El estudio de los diferentes climas organizativos y relacionales que se originan dentro de este tipo de organizaciones (centros educativos privados, concertados y públicos), como ya se lleva haciendo por distintos autores (Oswaldo, Aparecida, Elías \& Ferraz, 2012; Hinojo, Cáceres \& Raso, 2013; Pineda Alfonso, de Alba Fernández \& García Pérez, 2013).

- $\quad$ El fomento de la participación de las familias en las relaciones educativas y relacionales intercentros y entre Centros por medio de la creación de grupos de formación y trabajo desde las AMPAS con la asistencia necesaria de todos los implicados, centrados en la prevención y mejora de la convivencia.

- $\quad$ El diseño, desarrollo y mejora de programas formativos docentes ofertados por los distintos agentes y centrados en aspectos prácticos y experienciales multiculturales, sociales y psicopedagógicos con el fin de lograr una mayor calidad formativa.

- $\quad$ Encuentros con las distintas Administraciones, organismos y agentes educativos a nivel estatal, autonómico y local con el fin de aportar distintos puntos de vista en base a las necesidades de cada uno de estos actores, y 
generar así propuestas y acciones de mejora que satisfagan las necesidades reales de todos dentro de nuestro sistema educativo actual.

- Una revisión de las política de Admisión y libre elección de los Centros Educativos por parte de los padres y madres, en la que se pueda optar libremente por un centro específico en base a los valores y necesidades de cada familia, sin discriminar a ningún colectivo y siempre hasta cubrir las plazas disponibles.

\section{References:}

Anuario- Guía oficial de (1923). Ceuta: Ibero- africana- americana.

Anuario- Guía oficial de Marruecos- zona española posesiones españolas del norte de África y Golfo de Guinea- Gibraltar. (1924).Madrid: Iberoafricana- americana.

Anuario- Guía oficial de Marruecos y del África española (1927). Madrid: Ibero- africana- americana.

Anuario guía oficial de Marruecos y del África española (comercio y turismo) (1928). Madrid: Cía. Ibero-americana de publicaciones, S.A.

Archivo del Colegio La Inmaculada. Memorias de Cursos. Varias décadas. Publicación interna.

Archivo del Colegio Santa María Micaela. Crónicas 1935-1995.

Archivo histórico de la Universidad de Sevilla. Legajos 837, 1469, 1484, 3160, 3163.

Arnal y otros (1994). Investigación educativa. Fundamentos y metodología. Barcelona: Labor

Fox, D. (1981). El proceso de investigación en educación. Pamplona: EUNSA.

Hinojo Lucena, F.J., Cáceres Reché, M.P., \& Raso Sánchez, F. (2013). Análisis de los componentes organizativos de centros de formación profesional en España. Revista Latinoamericana De Ciencias Sociales, Niñez y Juventud, 11(2)

Ley Orgánica 8/1985, de 3 de julio, reguladora del Derecho a la Educación. (BOE núm. 159, de 04/07/1985).

Lozano, J.M. (1979). Biografía de la M. Alfonsa Cavín. Barcelona: Claret.

Marín Parra, V. (2012). La educación en Ceuta: 1912-1956. Ceuta: Archivo General Ciudad Autónoma de Ceuta.

Oswaldo, Y.C., Aparecida Dias, E., Elias Rueda Spers, V., \& Ferraz Filho, O. (2012). Impacto de los estresores laborales en los profesionales y en las organizaciones: Análisis de investigaciones publicadas. Invenio: Revista De Investigación Académica, (29), 67-80.

Pineda Alfonso, J.A., de Alba Fernández, N.d., \& García Pérez, F.F. (2013). El conflicto y la convivencia: Experimentación de un ámbito de 
investigación escolar y análisis del desarrollo profesional docente. (Tesis de doctorado) 